\title{
Interventions interactives dans les médias sociaux visant à promouvoir l'équité en matière de santé : vue d'ensemble des examens
}

\author{
V. Welch, Ph. D. (1); J. Petkovic, M. Sc. (2); J. Pardo Pardo, B. Sc. (2); T. Rader, MBSI (3); P. Tugwell, M.D. (1, 3, 4)
}

Cet article fait partie de notre série sur l'équité en santé.

Diffuser cet article sur Twitter

Cet article a fait l'objet d'une évaluation par les pairs.

\section{Résumé}

Introduction : L’utilisation des médias sociaux en matière de santé publique et de promotion de la santé est en croissance, car ces outils permettent d'éliminer les barrières géographiques et physiques à l'accès aux services et aux soins de santé. Cependant, ils sont une source potentielle d'inégalité en matière de santé car une partie de la population n’y a pas accès ou ne les utilise pas. Cet article a comme objectif d'évaluer les effets des interventions interactives dans les médias sociaux sur les résultats sanitaires, les changements de comportement et l'équité en matière de santé.

Méthodologie : Nous avons réalisé une synthèse rapide d'examens systématiques axés sur des interventions favorables aux interactions réciproques, que ce soit les forums de discussion, les réseaux sociaux (comme Facebook et Twitter), les blogues, les applications liées aux communautés électroniques ou le partage de contenu multimédia. Nous avons eu recours à une stratégie de recherche fine pour sélectionner les examens systématiques.

Résultats : Onze examens systématiques ont répondu à nos critères d'inclusion. La plupart des interventions visées par ces examens visaient des groupes de discussion en ligne ou des outils similaires. Ces interventions étaient isolées ou conjointes à d'autres. Sept examens ont fait état d'effets mixtes sur les résultats sanitaires et les comportements sains. On n’a constaté aucune analyse détaillée des caractéristiques liées aux inconvénients, notamment un statut socioéconomique plus faible ou l'âge. Par contre, certaines études ciblées ont montré que les interventions dans les médias sociaux étaient efficaces pour certaines populations précises sur le plan de l'âge, du statut socioéconomique, de l'ethnicité et du lieu de résidence. Quatre examens ont fait état d'avantages qualitatifs, comme la satisfaction, le recueil d'information et l'amélioration du soutien social.

Conclusion : Les interventions dans les médias sociaux se sont avérées efficaces pour certaines populations susceptibles d'être désavantagées (jeunes, aînés, personnes à faible statut socioéconomique et population rurale), ce qui prouve leur efficacité potentielle pour l'avancement de l'équité en matière de santé. La confirmation de cette efficacité nécessiterait toutefois une étude approfondie. Plusieurs examens ont soulevé la question de l'acceptabilité des interventions dans les médias sociaux. Seulement quatre études se sont penchées sur le niveau d'utilisation de l'intervention et elles ont toutes dévoilé un faible niveau d'utilisation. Il faudra réaliser d'autres travaux de recherche sur les plateformes des principaux médias sociaux actuels, particulièrement auprès des populations risquant d'être désavantagées, afin d'évaluer leurs effets sur les résultats sanitaires et l'équité en matière de santé.

Mots-clés : médias sociaux, populations désavantagées, santé publique, promotion de la santé, équité en santé
Points saillants

- Dans le domaine de la santé publique, on a davantage recours aux médias sociaux parce que les interventions peuvent transcender les barrières géographiques et physiques à l'accès aux services et aux soins de santé.

- Sept examens systématiques ont révélé des effets mixtes des interventions dans les médias sociaux sur l'amélioration des résultats sanitaires et les comportements sains.

- Certaines des études analysées ont fait état d'avantages découlant des interventions dans les médias sociaux, alors que d'autres n’ont révélé aucun changement ou ont conclu que les résultats étaient pires que ceux des autres types d'intervention.

- On en sait peu au sujet de la façon dont les caractéristiques de la conception et de l'exécution ainsi que l'intensité et la durée des interventions peuvent améliorer la santé, et on ne sait pas vraiment si elles sont susceptibles d'accroître les comportements négatifs et la stigmatisation ou encore d'aggraver certaines inégalités en matière de santé.

- Bon nombre d'études ont eu recours à des plateformes de médias sociaux élaborées par les chercheurs. On ignore les effets de l'utilisation de plateformes commerciales et de réseaux sociaux comme Facebook et Twitter sur l'efficacité des interventions en santé dans les médias sociaux.

Rattachement des auteurs :

1. Institut de recherche Élisabeth-Bruyère, Université d'Ottawa, Ottawa (Ontario), Canada

2. Centre pour la santé mondiale, Université d'Ottawa, Ottawa (Ontario), Canada

3. Institut de recherche de I'Hôpital d'Ottawa, Université d'Ottawa, Ottawa (Ontario), Canada

4. Département de médecine, Faculté de médecine, Université d'Ottawa, Ottawa (Ontario), Canada

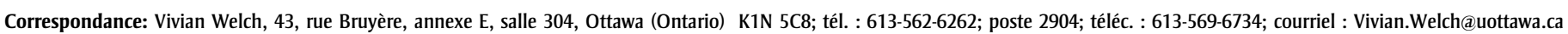




\section{Introduction}

L'utilisation des médias sociaux en matière de santé publique et de promotion de la santé est en croissance : $60 \%$ des départements aux États-Unis se servent d'au moins une application liée aux médias sociaux ${ }^{1}$; l'Agence de la santé publique du Canada (ASPC) est présente sur divers sites de médias sociaux, notamment Twitter (2 300 gazouillis et plus de 52000 abonnés en date du 14 janvier $2015)^{2}$ et Facebook (plus de 13000 « J'aime » en date du 14 janvier 2016) ${ }^{3}$ et 34 bureaux de santé publique sur 36 en Ontario ${ }^{4}$ utilisent les médias sociaux. Les médias sociaux sont une voie prometteuse pour les interventions en santé publique et l'ampleur de leur diffusion, car plus de $60 \%$ des adultes et $90 \%$ des jeunes ayant un accès Internet au Canada sont actifs sur au moins une de leurs plateformes $^{5,6}$.

On peut définir les médias sociaux par la mesure dans laquelle ils sont axés sur sept éléments constitutifs fonctionnels par rapport au degré d'interaction et de communication chez les utilisateurs, soit 1) l'identité : la mesure dans laquelle les utilisateurs se révèlent; 2) les conversations : la mesure dans laquelle les utilisateurs communiquent ensemble; 3) le partage : la mesure dans laquelle les utilisateurs échangent, distribuent et reçoivent du contenu; 4) la présence : la mesure dans laquelle les utilisateurs savent si les autres sont disponibles; 5) les relations : la mesure dans laquelle les utilisateurs interagissent; 6) la réputation : la mesure dans laquelle les utilisateurs connaissent bien la position sociale des autres et le contenu et 7) les groupes : la mesure dans laquelle les utilisateurs forment des communautés ${ }^{7}$.

Les éléments de preuve découlant des examens systématiques tendent à indiquer que les médias sociaux qui facilitent les échanges avec les autres utilisateurs, notamment au moyen de babillards, de bavardoirs et de sites de réseautage (comme Twitter et Facebook) améliorent efficacement les connaissances. Cependant, nous avons constaté que les effets sur les comportements liés à la santé (p. ex. tabagisme, alimentation et activité physique) et les résultats sanitaires (comme la perte de poids et la santé mentale) étaient tant positifs que négatifs $^{8-12}$. Bien que les interventions dans les médias sociaux dans ces examens aient en grande partie fait l'objet d'une évaluation auprès de populations instruites et ayant des revenus plus élevés, certaines études ont démontré également l'existence d'avantages pour les populations à faible revenu, les aînés, les jeunes et différents groupes ethnoculturels.

Par contre, les stratégies de santé publique qui s'appuient sur les médias de masse ont également le potentiel d'accroître les inégalités en matière de santé, à savoir des différences sur le plan des résultats sanitaires qui sont évitables et injustes ${ }^{13,14}$. Les différences d'accès à la technologie et les préférences et différences culturelles peuvent influer sur l'application et l'utilisation des interventions dans les médias sociaux, et entraîner des inégalités en matière de santé.

Cet article vise à évaluer les effets des interventions interactives dans les médias sociaux sur la communication au sujet des résultats sanitaires, du changement de comportement et de l'équité en matière de santé en faisant un survol des examens systématiques.

\section{Méthodes}

\section{Approche}

Nous avons défini la question de l'examen au moyen de l'approche axée sur la population, de l'intervention, des facteurs de comparaison et du résultat (outcome) $(\mathrm{PICO})^{15}$.

\section{Population ciblée}

Nous avons inclus les examens systématiques de toutes les populations exposées à des interventions dans les médias sociaux.

\section{Intervention}

Les médias sociaux désignent des « activités entre des gens regroupés en ligne qui échangent des renseignements à l'aide de dialogues facilitant la création et le partage de contenu sous forme de mots, d'images, de vidéos et d'enregistrements sonores ${ }^{16}$. Comme cela a été mentionné précédemment, il est possible de définir différents types de médias sociaux selon la mesure dans laquelle ils sont axés sur les sept éléments constitutifs fonctionnels ${ }^{7}$. Les médias sociaux permettent diverses activités : des forums de discussion, du réseautage social (p. ex. Facebook et Twitter), des blogues et des microblogues, la mise en signet et la diffusion (« partage ») de contenu média ${ }^{17}$. Pour qu'elle se distingue des autres programmes exécutés sur Internet et pour constituer une forme de média social dans le cadre de notre examen, une intervention devait offrir une composante interactive axée sur une communication bidirectionnelle entre les pairs ou entre le site Web et les utilisateurs.

Nous avons exclu les médias de masse et les formulaires unidirectionnels de communication au sujet de la santé (lorsqu'Internet ou les messages textes servaient à transmettre des messages sans composante interactive). Nous avons exclu également les interventions en matière de cybersanté qui utilisaient la technologie pour offrir des soins de santé (p. ex. utilisation d'une consultation à distance entre un patient et un fournisseur au moyen du réseau TéléAVC $)^{18,19}$. Nous avons exclu aussi les applications de téléphone intelligent qui n'étaient pas munies d'une composante interactive avec les autres utilisateurs (comme une fonction de rétroaction ou de suivi du poids sur un téléphone intelligent à des fins d'utilisation personnelle seulement, sans réaction ou rétroaction de la part des autres utilisateurs et des pairs).

\section{Comparateur}

Nous avons tenu compte des comparateurs de soins courants, sans intervention ou avec une autre méthode d'intervention pouvant avoir une composante de média social. Les soins courants pouvaient comprendre tout type de soins de santé ou toute activité de promotion de la santé. Nous nous sommes assuré de ce que le comparateur soit de large portée, de sorte qu'il soit possible d'en faire la comparaison avec toute autre méthode assurant la promotion de la santé ou la prestation de soins de santé pour le même état de santé.

\section{Résultats}

Nous avons pris en considération les examens systématiques qui rendaient compte d'au moins un des résultats primaires suivants : 
résultats physiques (p. ex. changement de poids et état fonctionnel), résultats en santé psychosociale (p. ex. qualité de vie et autoefficacité), satisfaction, changement de comportement et effets indésirables (p. ex. dépendance et dépression). Nous avons aussi recueilli et présenté les données sur les résultats secondaires, à savoir l'attitude et les connaissances. Nous avons documenté les mesures de processus, notamment la qualité de la communication, les connaissances, la portée, l'engagement et la fidélité de l'intervention (la mesure dans laquelle l'intervention a été mise en œuvre comme prévu) ${ }^{20}$.

Pour évaluer les résultats de l'équité en matière de santé, nous avons déterminé si les résultats étaient présentés séparément en fonction des caractéristiques liées aux privilèges et aux inconvénients. Nous avons également évalué si l'intervention visait une population désavantagée, ce qui pouvait améliorer l'équité en matière de santé. Nous avons eu recours à l'acronyme PROGRESSPlus pour établir ces caractéristiques. PROGRESS-Plus signifie Place (lieu) de résidence, Race, ethnicité, culture ou langue, Occupation (profession), Genre et sexe, Religion, Études, Statut socioéconomique, capital Social ou d'autres facteurs liés aux privilèges et aux inconvénients, comme l'âge (p. ex. enfants ou aînés), l'orientation sexuelle et l'état pathologique ${ }^{21}$.

\section{Plan d'étude}

Nous avons utilisé une synthèse rapide comme approche des examens systématiques. Une synthèse rapide donne un aperçu des éléments de preuve disponibles qui découle généralement de lignes directrices ou d'examens systématiques, en réponse à un besoin ou à une priorité spécifiques, dans un court délai, par un utilisateur des connaissances $^{22,23}$. Un modèle de protocole a été mis au point, puis transmis à l'ASPC en tant qu'énoncé de travail (fourni sur demande). Nous avons défini l'examen systématique comme une synthèse systématique et claire des études admissibles, avec des méthodes claires et une stratégie de recherche explicite. Nous avons pris en compte des examens d'essais contrôlés randomisés, d'études non randomisées et d'études qualitatives.
Méthodes de recherche en vue du choix des études

Nous avons conçu une stratégie fine de recherche en vue de l'extraction des examens systématiques des bases de données bibliographiques électroniques. D’après notre utilisateur de connaissances, il était préférable d'éviter de faire une recherche dans la littérature grise en raison des contraintes de temps. Pour extraire les examens systématiques, nous avons utilisé le filtre de Montori, un filtre validé de modèle d'étude des examens systématiques $^{24}$. Aucune restriction sur le plan des dates ou des langues n’a été appliquée.

Le 27 février 2014, nous avons dénombré 4580 articles découlant des bases de données suivantes :

- MEDLINE par OVID $\left(1946^{*}\right.$ au 27 février 2014);

- PsycINFO par OVID (PsycINFO, 1806 à la troisième semaine de février 2014);

- Bibliothèque Cochrane par Wiley (deuxième de 12 numéros, 2014), dont la Database of Abstracts of Reviews of Effects (DARE) de Cochrane, la Cochrane Database of Systematic Reviews (CDSR), l'évaluation des technologies de la santé (ETS) et l'Economic Evaluations Database (EED) en vue de la numérisation des listes de référence des examens systématiques pertinents;

- PubMed par la National Library of Medicine, recherche des « articles connexes » dans PubMed à l'aide de quatre examens systématiques pertinents en tant qu'articles sources, 27 février 2014;

- Bibliothèque Campbell (recherche de tous les numéros à la main, 2004 à aujourd'hui).

La stratégie de recherche a été conçue dans OVID MEDLINE par un bibliothécaire scientifique (TR) et vérifiée par un autre membre de l'équipe (JPP) conformément aux lignes directrices des stratégies de recherche électroniques revues par les pairs (PRESS) ${ }^{25}$. La stratégie a ensuite été adaptée aux autres bases de données. Les stratégies de recherche complètes peuvent être fournies sur demande.
La recherche dans toutes les bases de données s'est faite de leur date de départ au 27 février 2014. Les doublons ont été supprimés à l'aide d'EndNote, et le nombre final de citations s'est chiffré à 4102 .

\section{Critères d'inclusion}

Nous avons tenu compte des examens dans le cadre desquels avaient été évalués les effets des interventions en promotion de la santé dans les réseaux sociaux (description des interventions admissibles décrites au tableau 1) sur les comportements liés à la santé ou les résultats sanitaires.

\section{Extraction des données}

Nous avons extrait les données portant sur les éléments suivants :

- Description de l'intervention;

- Comparateur;

- Résultats;

- Critères d'exclusion des examens;

- Nombre d'études incluses;

- Nombre de participants au sein des groupes d'intervention et de contrôle (personnes inscrites et ayant participé aux séances);

- Pays;

- Description de la population, notamment l'âge médian et le pourcentage de femmes;

- Description de la population, analyse ou interprétation selon l'acronyme PROGRESS-Plus;

- Résultat - résultat sommaire et résultat quantitatif regroupés (le cas échéant);

- Utilisation des médias sociaux (mesure dans laquelle les participants ont eu recours à l'intervention), portée de l'intervention et activités visant à accroître l'engagement des participants à l'égard de l'intervention (p. ex. recours aux services d'un modérateur);

- Variables confusionnelles;

- Effets indésirables;

- Risque de parti pris;

- Pertinence par rapport aux populations visées par PROGRESS-Plus;

- Score AMSTAR.

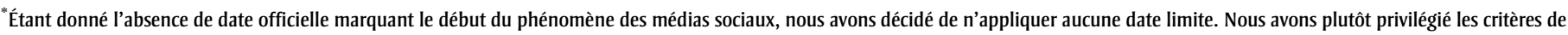
recherche qui décrivaient l'intervention dans les médias sociaux et avons extrait les documents pertinents, abstraction faite de la date de publication. 
TABLEAU 1

Définition des interventions dans les médias sociaux

\begin{tabular}{|c|c|c|}
\hline Format des médias sociaux & Inclus & Exclus \\
\hline $\begin{array}{l}\text { Blogues et microblogues (p. ex. } \\
\text { Twitter) }\end{array}$ & $\begin{array}{l}\text { Si l'intervention comprend une interaction } \\
\text { à voies multiples }\end{array}$ & $\begin{array}{l}\text { Messages et articles unidirectionnels ou contact direct avec } \\
\text { un fournisseur de soins de santé }\end{array}$ \\
\hline $\begin{array}{l}\text { Communautés de contenu (p. ex. } \\
\text { YouTube et Pinterest) }\end{array}$ & $\begin{array}{l}\text { Si l'intervention comprend une interaction } \\
\text { à voies multiples }\end{array}$ & $\begin{array}{l}\text { Messages et articles unidirectionnels ou contact direct avec } \\
\text { un fournisseur de soins de santé }\end{array}$ \\
\hline $\begin{array}{l}\text { Groupes de discussion (p. ex. } \\
\text { bavardoirs, babillards en ligne et } \\
\text { forums de discussion) }\end{array}$ & $\begin{array}{l}\text { Groupes de discussion ou babillards } \\
\text { synchrones ou asynchrones }\end{array}$ & $\begin{array}{l}\text { Messages et articles unidirectionnels ou contact direct avec } \\
\text { un fournisseur de soins de santé }\end{array}$ \\
\hline Courriels & $\begin{array}{l}\text { Serveurs de liste qui favorisent la communication, la } \\
\text { discussion et I'accès aux comptes rendus des discussions à } \\
\text { des fins d'examen et de rétroaction }\end{array}$ & Courriels unidirectionnels (p. ex. rappels) \\
\hline Applications mobiles & $\begin{array}{l}\text { Applications qui favorisent la communication } \\
\text { et les échanges avec un groupe de personnes }\end{array}$ & $\begin{array}{l}\text { Applications qui permettent à une personne d'effectuer un } \\
\text { suivi de ses progrès et de les surveiller (p. ex. perte de poids } \\
\text { et glycémie) sans qu'il y ait une composante sociale ou des } \\
\text { applications servant à communiquer avec un fournisseur } \\
\text { de soins de santé }\end{array}$ \\
\hline SMS et messages textes & $\begin{array}{l}\text { Si le message reste affiché pour que les autres puissent le } \\
\text { consulter }\end{array}$ & $\begin{array}{l}\text { Messages textes unidirectionnels (p. ex. rappels) ou messages } \\
\text { textes auxquels un fournisseur de soins de santé ou des } \\
\text { chercheurs répondent ou au sujet desquels ils présentent une } \\
\text { rétroaction }\end{array}$ \\
\hline Jeux en ligne & $\begin{array}{l}\text { S'il y a une communication entre de nombreux joueurs (et } \\
\text { s'il y a un résultat en matière de santé) }\end{array}$ & Jeux en ligne sans composante sociale et de santé \\
\hline $\begin{array}{l}\text { Réseaux sociaux virtuels (p. ex. } \\
\text { Facebook) }\end{array}$ & $\begin{array}{l}\text { Si l'intervention comprend une interaction } \\
\text { à voies multiples }\end{array}$ & $\begin{array}{l}\text { Messages et articles unidirectionnels ou contact direct avec un } \\
\text { fournisseur de soins de santé }\end{array}$ \\
\hline Pages Web et wikis & $\begin{array}{l}\text { Si le site Web ou le wiki favorise l'interaction } \\
\text { à voies multiples }\end{array}$ & Communication unidirectionnelle (p. ex. éducation) \\
\hline
\end{tabular}

\section{Évaluation de la qualité des examens}

Nous avons eu recours à l'outil AMSTAR (http://amstar.ca/Amstar_Checklist.php) pour évaluer la qualité des examens systématiques ${ }^{26}$. Nous avons estimé que les examens systématiques étaient de bonne qualité lorsqu'ils abordaient les 11 éléments figurant sur la liste de vérification d'AMSTAR.

\section{Méthodes de synthèse}

Les interventions et les populations étaient trop hétérogènes pour que les résultats soient regroupés. Nous avons résumé de façon descriptive les effets sur les résultats importants pour les participants pour chaque type d'intervention ainsi que sur les résultats liés à la procédure, notamment en ce qui concerne la fidélité de l'intervention et la portée comme le niveau de l'engagement (si la mesure existe) à l'aide de l'ampleur de l'effet (si cette donnée est fournie). Nous avons vérifié la mesure dans laquelle les études primaires visées par les examens systématiques admissibles se chevauchaient. Les résultats dichotomiques sont présentés sous forme de risques relatifs, tandis que les résultats continus pondérés expriment les différences. On présente les résultats regroupés découlant des examens systématiques ayant combiné les résultats de façon statistique.

\section{Évaluation de l'impact sur l'équité en matière de santé}

Nous avons eu recours à l'Évaluation de l'impact sur l'équité en matière de santé (EIES), outil conçu par le ministère de la Santé et des Soins de longue durée (MSSLD) de l'Ontario (consulter le site http://www. health.gov.on.ca/fr/pro/programs/heia/), pour évaluer les effets prévisibles et imprévisibles probables pour les populations prioritaires (il est possible de se procurer une grille remplie auprès des auteurs sur demande). Pour porter un jugement au sujet des effets imprévisibles et des stratégies d'atténuation, deux membres de l'équipe de chercheurs (JP et VW) ont examiné les données sur l'accès à Internet ainsi que les obstacles et les éléments facilitateurs connus propres à des souspopulations précises en fonction de l'expertise de l'équipe sur le plan de l'examen des effets des interventions sur l'équité en matière de santét $^{21,27}$. L'EIES vise à permettre de déterminer l'impact d'un programme, d'une politique ou d'une initiative sur différents groupes de population, et il est d'abord axé sur les barrières à l'accès aux programmes ${ }^{28}$. Nous avons eu recours aux données sur l'accès aux plateformes de médias sociaux et leur utilisation découlant de l'Enquête canadienne sur l'utilisation de l'Internet (ECUI) pour mesurer l'accès à Internet et les comportements en matière d'utilisation d'Internet (il est possible de consulter les résultats de l'enquête sur demande).

\section{Résultats}

\section{Affichage des résultats de la recherche}

La stratégie de recherche a permis de détecter 4103 dossiers à la suite de la suppression des doublons. Le tri des résumés et des titres a permis d'exclure 3957 dossiers et d'extraire 146 articles en vue de l'examen de texte intégral. Nous avons exclu 135 examens systématiques parce que les interventions étaient axées sur la communication unidirectionnelle (p. ex. rappels de rendez-vous) ou sur le 
recours à Internet pour des traitements (p. ex. thérapie cognitive sur Internet) ou pour les messages unidirectionnels de sensibilisation ou d'information n'entraînant aucune interaction entre les utilisateurs ni aucune production de contenu par les utilisateurs (voir la figure 1). Un tableau des études exclues est fourni sur demande.

Onze examens systématiques ont été intégrés au final dans notre synthèse (le tableau 2 présente les caractéristiques de ces études).

\section{Description des examens}

La plupart des examens systématiques portaient sur des babillards en ligne ou des stratégies du même type, que ce soit des interventions autonomes ou conjointes à d'autres interventions. Seulement trois examens englobaient des études dans le cadre desquelles on s'était servi des outils de médias sociaux les plus courants, à savoir Facebook (19 études uniques) et Twitter $(9 \text { études uniques })^{8,9,30}$. Bien qu'elles aient été visées par les critères de recherche, aucune des interventions des examens systématiques n'a porté sur des applications de téléphone cellulaire.

Dans les études antérieures mentionnées dans les examens, la plupart des babillards étaient des espaces «fermés » où les participants aux études étaient les seules personnes qui y avaient accès (il n'y avait aucune interaction avec les communautés ou les gens de l'extérieur). À titre d'exemple, dans l'examen de Chang et coll. ${ }^{9}$ sur la gestion du poids en ligne par les médias sociaux, $85 \%$ des études ont privilégié un site Web fermé et conçu par des chercheurs.

Dans les études où les médias sociaux faisaient partie intégrante d'une intervention complexe, les auteurs avaient mis l'accent sur le maintien d'un engagement avec les autres composantes de l'intervention afin de présenter une rétroaction au sujet de l'intervention en soi ainsi qu'un espace où formuler des questions et obtenir les réponses. Par exemple, dans l'examen de Williams et coll. $^{30}$, dans le cadre des 16 études, les médias sociaux formaient l'une des

FIGURE 1

Organigramme PRISMA de $2009^{29}$ illustrant le processus de sélection en vue de l'établissement des examens systématiques pertinents

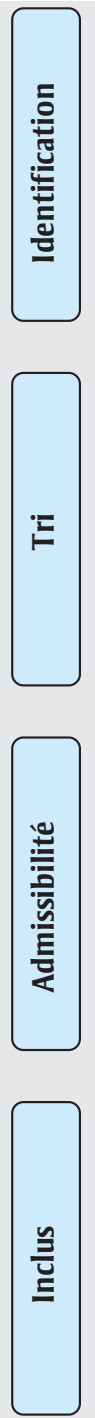

Dossiers détectés lors de la recherche dans les bases de données $(n=4580)$
Dossiers supplémentaires détectés par l'entremise d'autres sources

$$
(n=0)
$$

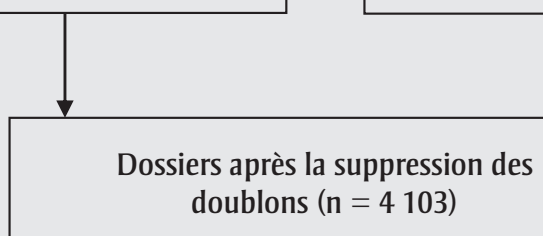

\section{(n)}
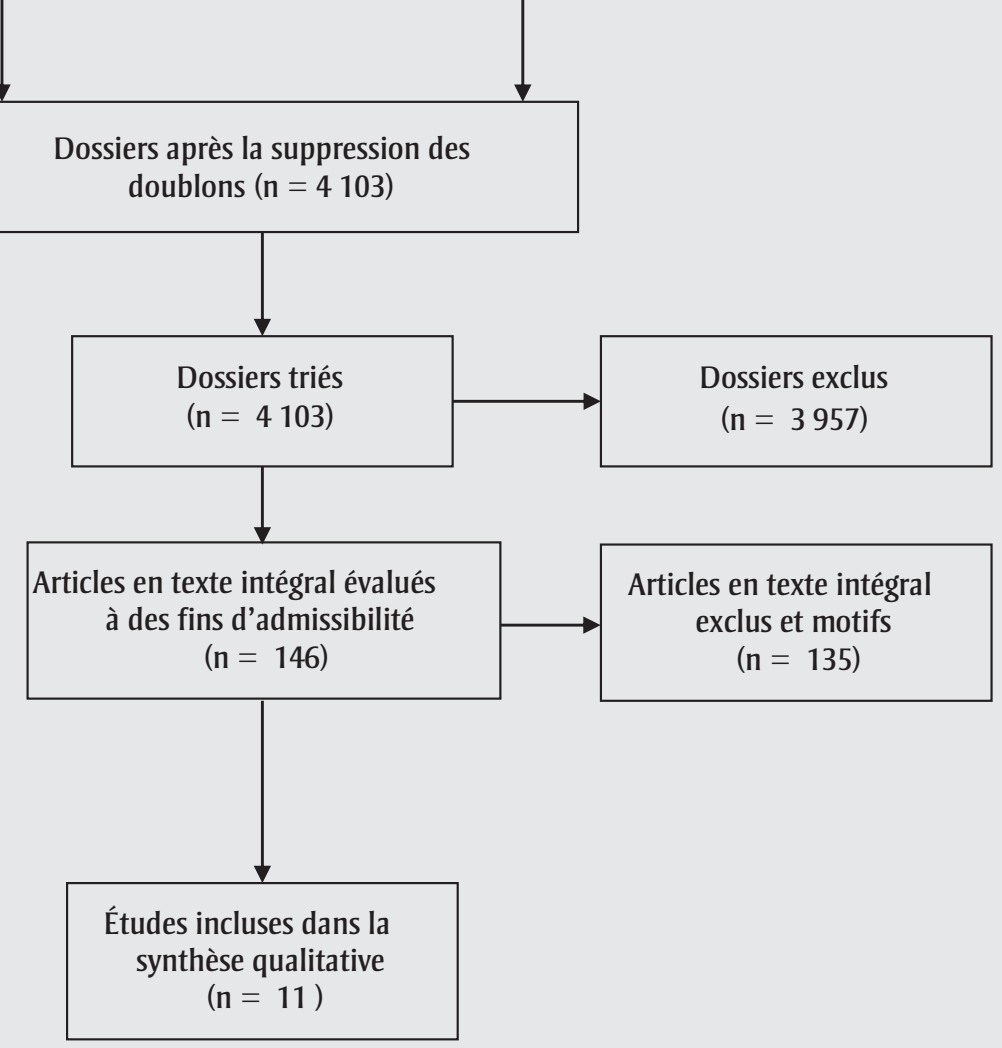
TABLEAU 2

Caractéristiques des études incluses (interventions, populations et résultats)

\begin{tabular}{|c|c|c|c|c|c|c|c|c|}
\hline Référence & Population & Interventions & $\begin{array}{c}\text { Critères } \\
\text { d'admissibilité }\end{array}$ & $\begin{array}{l}\text { Résultat cible } \\
\text { (durée du } \\
\text { suivi) }\end{array}$ & $\begin{array}{l}\text { Nombre } \\
\text { d'études } \\
\text { (nombre } \\
\text { d'études } \\
\text { ayant des } \\
\text { composantes } \\
\text { de médias } \\
\text { sociaux) }\end{array}$ & $\begin{array}{c}\text { Types } \\
\text { d'études } \\
\text { admissibles }\end{array}$ & $\begin{array}{c}\text { Pays } \\
\text { (nombre } \\
\text { d'études) }\end{array}$ & $\begin{array}{c}\text { Score à } \\
\text { I'outil } \\
\text { AMSTAR }\end{array}$ \\
\hline $\begin{array}{l}\text { Chang et } \\
\text { coll., } 2012^{9}\end{array}$ & $\begin{array}{l}\text { Personnes de tous les } \\
\text { âges qui utilisent les } \\
\text { médias sociaux pour } \\
\text { la gestion du poids }\end{array}$ & $\begin{array}{l}\text { La plupart des } \\
\text { interventions ont pris } \\
\text { la forme de babillards } \\
\text { ou de bavardoirs } \\
\text { conçus par les } \\
\text { chercheurs; Facebook } \\
\text { a été utilisé pour } \\
\text { deux études, alors } \\
\text { que Twitter l'a été } \\
\text { pour une étude. }\end{array}$ & $\begin{array}{l}\text { Le signalement des } \\
\text { résultats liés au poids } \\
\text { comprend une } \\
\text { composante de } \\
\text { média social } \\
\text { (application Web } \\
\text { favorisant } \\
\text { l'interaction dans } \\
\text { une communauté } \\
\text { virtuelle). }\end{array}$ & $\begin{array}{l}\text { Gestion du poids } \\
\text { (6 à } 12 \text { mois) }\end{array}$ & 20 (20) & ECR & $\begin{array}{l}\text { États-Unis } \\
(n=14), \\
\text { Australie } \\
(n=3), \\
\text { Canada } \\
(n=2) \\
\text { et Royaume- } \\
\text { Uni ( } n=1)\end{array}$ & 5 \\
\hline $\begin{array}{l}\text { Eysenbach } \\
\text { et coll., } \\
2004^{34}\end{array}$ & $\begin{array}{l}\text { Divers groupes, p. ex. } \\
\text { les femmes } \\
\text { enceintes, les } \\
\text { fournisseurs de soins } \\
\text { pour les personnes } \\
\text { atteintes de la } \\
\text { maladie d'Alzheimer, } \\
\text { les adultes atteints } \\
\text { de diabète, les } \\
\text { personnes atteintes } \\
\text { du sida, les jeunes } \\
\text { mères célibataires et } \\
\text { les fumeurs adultes }\end{array}$ & $\begin{array}{l}\text { Communautés } \\
\text { virtuelles, forums de } \\
\text { discussion sur le Web, } \\
\text { bavardoirs, liste } \\
\text { d'envoi et babillards } \\
\text { vocaux qui englobent } \\
\text { une interaction entre } \\
\text { pairs }\end{array}$ & $\begin{array}{l}\text { Les interventions } \\
\text { devaient prendre la } \\
\text { forme d'une } \\
\text { communauté } \\
\text { virtuelle ou avoir une } \\
\text { composante de } \\
\text { communauté } \\
\text { virtuelle (groupe de } \\
\text { personnes qui } \\
\text { interagissent } \\
\text { publiquement au } \\
\text { moyen d'un réseau } \\
\text { de communication } \\
\text { informatique ou d'un } \\
\text { autre outil } \\
\text { informatique), être } \\
\text { axées en grande } \\
\text { partie sur les } \\
\text { questions liées à la } \\
\text { santé ou aux soins de } \\
\text { santé, et comporter } \\
\text { des résultats } \\
\text { touchant les } \\
\text { connaissances et la } \\
\text { santé ainsi que des } \\
\text { résultats } \\
\text { psychologiques ou } \\
\text { sociaux. Le recours } \\
\text { aux services de santé } \\
\text { se rattachait à un } \\
\text { groupe de contrôle. }\end{array}$ & $\begin{array}{l}\text { Résultats } \\
\text { sociaux et en } \\
\text { matière de santé } \\
\text { (10 semaines à } \\
12 \text { mois) }\end{array}$ & 38 (38) & ECR et CAA & Non signalé & 6 \\
\hline $\begin{array}{l}\text { Griffiths, } \\
2009^{39}\end{array}$ & $\begin{array}{l}\text { Adultes et } \\
\text { adolescents, certains } \\
\text { participants atteints } \\
\text { de cancer, de } \\
\text { dépression, de } \\
\text { maladies chroniques } \\
\text { ou de VIH, et } \\
\text { fournisseurs de soins }\end{array}$ & $\begin{array}{l}\text { Groupes de soutien } \\
\text { sur Internet (p. ex. } \\
\text { babillards, bavardoirs } \\
\text { ou listes d'envoi } \\
\text { utilisés seuls ou en } \\
\text { combinaison l'un avec } \\
\text { l'autre) }\end{array}$ & $\begin{array}{l}\text { Les interventions se } \\
\text { traduisaient } \\
\text { notamment par un } \\
\text { groupe de soutien en } \\
\text { ligne entre pairs, et } \\
\text { leur résultat était axé } \\
\text { sur la dépression. On } \\
\text { y trouvait un groupe } \\
\text { de soutien unipolaire } \\
\text { sur Internet pour les } \\
\text { personnes atteintes } \\
\text { de dépression. }\end{array}$ & $\begin{array}{l}\text { Dépression } \\
\text { (12 semaines } \\
\text { à } 12 \text { mois) }\end{array}$ & $28(10)$ & $\begin{array}{l}\text { ECR, CAA, } \\
\text { série de cas, } \\
\text { étude } \\
\text { transversale et } \\
\text { SCI }\end{array}$ & $\begin{array}{l}\text { États-Unis } \\
(n=23) \text {, } \\
\text { Europe } \\
(n=4) \\
\text { et Australie } \\
(n=1)\end{array}$ & 9 \\
\hline
\end{tabular}


TABLEAU 2 (suite)

Caractéristiques des études incluses (interventions, populations et résultats)

\begin{tabular}{|c|c|c|c|c|c|c|c|c|}
\hline Référence & Population & Interventions & $\begin{array}{c}\text { Critères } \\
\text { d'admissibilité }\end{array}$ & $\begin{array}{l}\text { Résultat cible } \\
\text { (durée du } \\
\text { suivi) }\end{array}$ & $\begin{array}{l}\text { Nombre } \\
\text { d'études } \\
\text { (nombre } \\
\text { d'études } \\
\text { ayant des } \\
\text { composantes } \\
\text { de médias } \\
\text { sociaux) } \\
\end{array}$ & $\begin{array}{c}\text { Types } \\
\text { d'études } \\
\text { admissibles }\end{array}$ & $\begin{array}{c}\text { Pays } \\
\text { (nombre } \\
\text { d'études) }\end{array}$ & $\begin{array}{l}\text { Score à } \\
\text { I'outil } \\
\text { AMSTAR }\end{array}$ \\
\hline $\begin{array}{l}\text { Lai et coll., } \\
2014^{31}\end{array}$ & Surtout des adultes & $\begin{array}{l}\text { Mesures de } \\
\text { prévention du suicide } \\
\text { sur le Web, } \\
\text { notamment toute } \\
\text { intervention sur } \\
\text { Internet (p. ex. } \\
\text { thérapie cognitivo- } \\
\text { comportementale, } \\
\text { groupes de soutien en } \\
\text { ligne et babillard) }\end{array}$ & $\begin{array}{l}\text { Stratégies de } \\
\text { prévention du suicide } \\
\text { sur le Web et examen } \\
\text { de l'efficacité, des } \\
\text { avantages ou des } \\
\text { difficultés de } \\
\text { l'intervention }\end{array}$ & $\begin{array}{l}\text { Idées suicidaires } \\
\text { (6 semaines à } \\
12 \text { mois) }\end{array}$ & $15(4)$ & $\begin{array}{l}\text { ECR, CAA, } \\
\text { série de cas, } \\
\text { étude de } \\
\text { cohorte, étude } \\
\text { transversale, } \\
\text { rapports } \\
\text { qualitatifs et } \\
\text { rapports } \\
\text { descriptifs }\end{array}$ & États-Unis & 6 \\
\hline $\begin{array}{l}\text { Moorhead } \\
\text { et coll., } \\
2013^{8}\end{array}$ & $\begin{array}{l}\text { Personnes de tous } \\
\text { âges, allant d'enfants } \\
\text { d'âge scolaire à des } \\
\text { aînés, et dont le } \\
\text { niveau d'études, le } \\
\text { statut } \\
\text { socioéconomique et } \\
\text { l'ethnicité varient }\end{array}$ & $\begin{array}{l}\text { Communication entre } \\
\text { le grand public, les } \\
\text { patients et les } \\
\text { professionnels de la } \\
\text { santé au sujet des } \\
\text { questions de santé au } \\
\text { moyen des médias } \\
\text { sociaux (Facebook } \\
\text { [n }=13 \text { ], blogues } \\
\text { [n }=13 \text { ], Twitter } \\
\text { [n }=8 \text { ], YouTube } \\
\text { [n }=7 \text { ], Myspace } \\
\text { [n }=5], \text { Patients } \\
\text { LikeMe [n = 4] } \\
\text { et autres types de } \\
\text { médias sociaux } \\
\text { [n }=53] \text { ]) }\end{array}$ & $\begin{array}{l}\text { Les interventions } \\
\text { devaient être axées } \\
\text { principalement sur } \\
\text { l'ensemble des } \\
\text { interactions au sein } \\
\text { du grand public et } \\
\text { entre ce dernier, les } \\
\text { patients et les } \\
\text { professionnels de la } \\
\text { santé au sujet des } \\
\text { questions de santé } \\
\text { au moyen des } \\
\text { médias sociaux, } \\
\text { notamment en ce qui } \\
\text { concerne les } \\
\text { utilisations, les } \\
\text { avantages ou les } \\
\text { limites des médias } \\
\text { sociaux pour la } \\
\text { communication sur } \\
\text { la santé. }\end{array}$ & $\begin{array}{l}\text { Utilisation, } \\
\text { avantages et } \\
\text { limites des } \\
\text { médias sociaux } \\
\text { (non signalé) }\end{array}$ & $98(98)$ & $\begin{array}{l}\text { ECR, analyses } \\
\text { de réseau, } \\
\text { étude } \\
\text { transversale, } \\
\text { rapports } \\
\text { qualitatifs, } \\
\text { rapports } \\
\text { descriptifs et } \\
\text { analyses de } \\
\text { données } \\
\text { secondaires }\end{array}$ & $\begin{array}{l}\text { Surtout des } \\
\text { personnes à } \\
\text { revenu élevé } \\
\text { (non } \\
\text { signalé) }\end{array}$ & 5 \\
\hline
\end{tabular}


TABLEAU 2 (suite)

Caractéristiques des études incluses (interventions, populations et résultats)

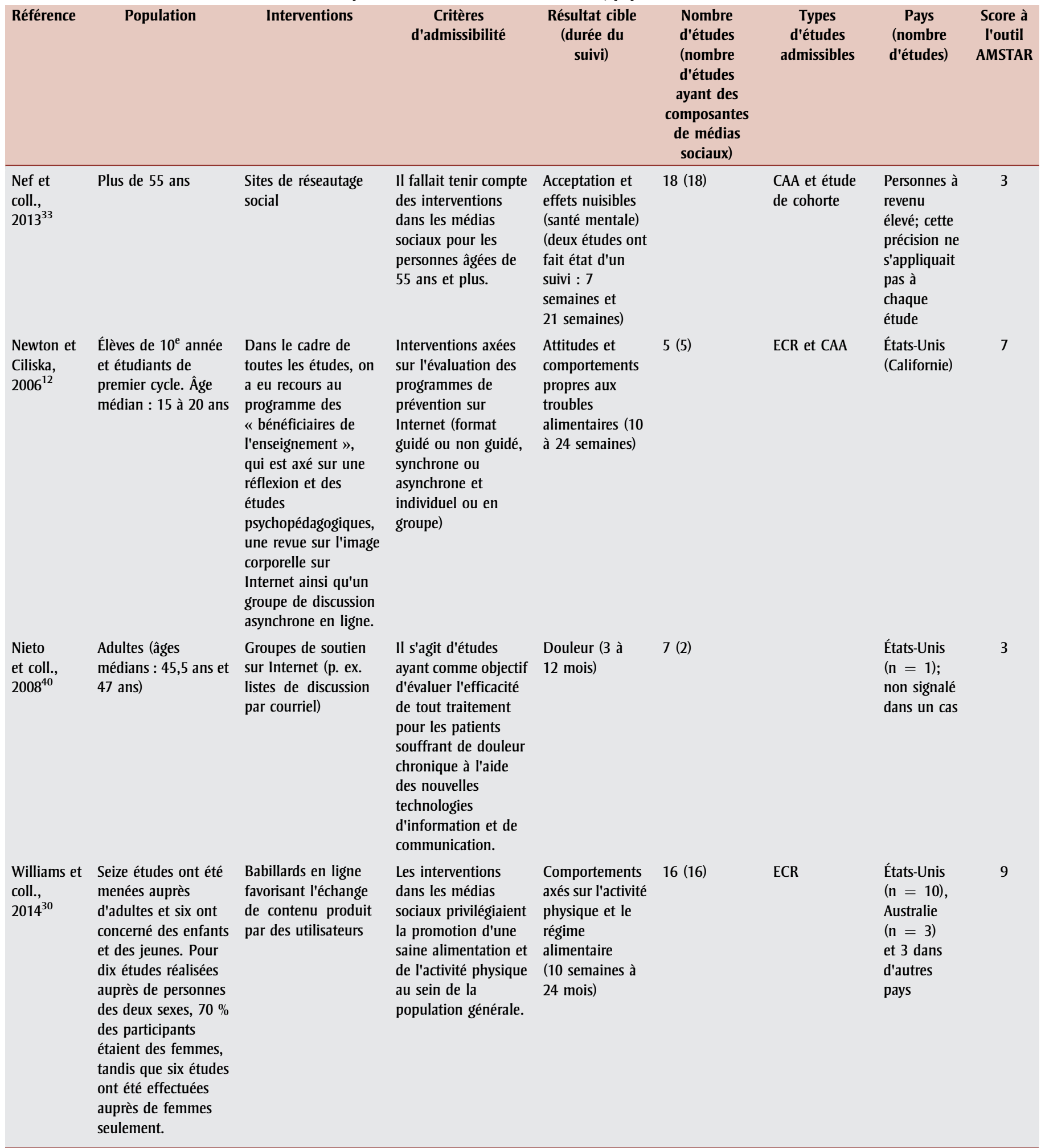

Abréviations : CAA, contrôle avant-après; ECR, essai clinique randomisé; SCI, séries chronologiques interrompues.

composantes d'une intervention complexe, tandis que les autres composantes étaient des sites Web présentant des fiches signalétiques et des renseignements ou des échanges par courriel dirigés par des thérapeutes.
Dans les 11 examens systématiques en question, les populations étaient diversifiées sur le plan de l'âge (enfants, jeunes, adultes et aînés), de l'état sous-jacent (p. ex. cancer, maladie cardiovasculaire, asthme, dépression et troubles de l'alimentation) ainsi que du sexe et du genre (il n'y avait aucun critère d'exclusion lié au sexe et au genre). Un examen systématique important constitué de 98 études sur les interventions dans les médias sociaux pour un large éventail d'activités de promotion de la santé ciblait 
des populations diversifiées par rapport à l'ethnicité, au statut socioéconomique, à l'âge et aux etudes ${ }^{8}$.

\section{Effets sur les interventions dans les médias sociaux}

Il a été impossible de combiner les résultats pour produire des méta-analyses groupées. Les résultats de chaque examen sont résumés dans le tableau 3. Nous avons rendu compte de l'ampleur de l'effet ainsi que des intervalles de confiance (IC) lorsqu'ils étaient disponibles.

Sur les 11 examens pris en compte, sept ont signalé des effets mixtes sur les résultats sanitaires et les comportements liés à la santé (voir les détails ci-dessous). Les quatre autres ont fait état d'avantages, comme la satisfaction, la détection de renseignements et le soutien social ${ }^{8,31-33}$.

\section{Connaissances}

L'examen réalisé par Newton et Ciliska ${ }^{12}$ (score de 7 à l'outil AMSTAR) a rendu compte des résultats en matière de connaissances et indiqué « une augmentation statistiquement significative des connaissances » au sujet des attitudes et des comportements liés aux modes de vie sains.

\section{Perte de poids}

Un examen effectué par Williams et coll. ${ }^{30}$ (score de 9 à l'outil AMSTAR) a regroupé neuf études sur les interventions dans les médias sociaux visant la perte de poids et n'a signalé aucune différence entre les groupes des médias sociaux et les groupes témoins (poids : 0,00 kg; IC à $95 \%$ : $-0,19$ à 0,19). Cependant, un autre examen, fait par Chang et coll. ${ }^{9}$ (score de 5 à l'outil AMSTAR), a tenu compte de cinq des études utilisées par Williams et coll. ${ }^{30}$ et conclu que neuf études avaient indiqué une perte de poids plus importante au moyen de l'intervention dans les médias sociaux qu'au sein du groupe témoin, mais que quatre études n'avaient démontré aucun effet et que deux avaient révélé une perte de poids moins élevée au sein du groupe des médias sociaux que chez les personnes ayant des interventions en face à face. Un troisième examen, réalisé par Eysenbach et coll. ${ }^{34}$ (score de 6 à l'outil AMSTAR), a permis de constater des « effets mixtes, mais en grande partie négligeables » des médias sociaux sur les résultats liés au poids.

Changement de comportement - activité physique

L'examen de Chang et coll. ${ }^{9}$ (score de 5 à l'outil AMSTAR) a signalé que deux études indiquaient une hausse de l'activité physique autodéclarée, mais que trois études ne démontraient aucune différence sur le plan de l'activité physique. Williams et coll. ${ }^{30}$ (score de 9 à l'outil AMSTAR) ont réalisé une méta-analyse de 12 études et fait état d'une augmentation de l'activité physique au sein du groupe des médias sociaux (différence des moyennes standardisées [DMS] : 0,13; IC à $95 \%:-0,04$ à 0,30).

\section{Changement de comportement - régime}

Un examen réalisé par Medina et coll. ${ }^{32}$ (score de 3 à l'outil AMSTAR) a traité des plateformes de médias sociaux en ligne à l'intention des personnes ayant une maladie cardiovasculaire et décrit l'amélioration du régime et de la qualité de vie chez celles qui utilisaient ces plateformes. L'examen de Chang et coll. ${ }^{9}$ (score de 5 à l'outil AMSTAR) a tenu compte d'une étude selon laquelle il n'y avait « aucune différence statistiquement significative » de l'indice de masse corporelle (IMC), du rapport taille/hanches (RTH), de la pression sanguine ou du cholestérol à la suite d'une intervention alimentaire.

Changement de comportement - tabagisme L'examen effectué par Eysenbach et coll. ${ }^{34}$ (score de 6 à l'outil AMSTAR) a signalé un taux de renoncement au tabac plus élevé au sein d'un groupe de soutien en ligne entre pairs qu'au sein d'un groupe sans soutien entre pairs. Par contre, le taux au sein du groupe qui bénéficiait d'un soutien en ligne entre pairs et d'une intervention psychopédagogique était semblable à celui du groupe qui recevait uniquement une intervention psychopédagogique.

\section{Utilisation des soins de santé}

Selon une étude abordée dans l'examen de Medina et coll. $^{32}$ (score de 3 à l'outil AMSTAR), le nombre de visites chez le médecin « a diminué de façon modérée » à la suite d'une intervention au sein d'un groupe de soutien en ligne où il y avait un modérateur. Cependant, cette tendance n'a pas persisté au cours de la deuxième phase à la suite du retrait du modérateur ${ }^{32}$. L'examen d'Eysenbach et coll. ${ }^{34}$ (score de 6 à l'outil AMSTAR) a porté sur trois études qui portaient sur l'utilisation des soins de santé. Une étude visée par cet examen a indiqué une diminution du nombre d'appels téléphoniques chez le médecin à la suite de l'intervention dans les médias sociaux, alors qu'une autre a plutôt démontré une augmentation du nombre d'appels téléphoniques chez des fournisseurs (l'ampleur de l'effet n'était pas précisée, mais les auteurs ont indiqué la formule $p<0,05$ ).

\section{Résultats en santé mentale}

L'examen de Lai et coll. ${ }^{31}$ (score de 6 à l'outil AMSTAR) concernant quatre études ayant des composantes de médias sociaux a permis de conclure que la thérapie cognitivocomportementale sur Internet jumelée à des interventions dans les médias sociaux (babillard électronique ou groupes de soutien) avait réduit les idées suicidaires (variation de l'ampleur de l'effet : $d=0,04$ à 0,45).

L'examen réalisé par Hong et coll. ${ }^{35}$ (score de 3 à l'outil AMSTAR) a porté sur les effets des groupes sur Internet sur la dépression chez les survivants du cancer, principalement ceux du cancer du sein. Les participants ont eu une opinion positive des outils sur Internet, et la plupart des études ont indiqué des effets positifs des groupes de médias sociaux. Cependant, dans le cas des quelques interventions qui ont été comparées à un autre type de programme (p. ex. programme en face à face), l'intervention dans les médias sociaux a eu des résultats semblables ou a été moins efficace (p. ex. une étude a fait état de taux de dépression plus élevés au sein du groupe des médias sociaux qu'au sein du groupe en face à face).

\section{Effets nuisibles potentiels}

Les effets nuisibles ou indésirables n'ont été quantifiés dans aucun des examens systématiques. La plupart des études visées par les examens ont signalé des taux de diminution d'au moins $20 \%{ }^{9,30}$. Les motifs justifiant ces taux d'attrition n'ont pas été analysés dans les études primaires.

Les examens ont décrit les effets nuisibles possibles, notamment l'absence de détection de symptômes, les préoccupations relatives à la qualité des soins, le stress accru, 
TABLEAU 3

Résultats des examens systématiques inclus

\begin{tabular}{|c|c|c|c|c|}
\hline Référence & Résultats souhaitables & Effets nuisibles ou limites & $\begin{array}{c}\text { Utilisation et atteinte d'un } \\
\text { engagement }\end{array}$ & Conclusions de l'examen \\
\hline $\begin{array}{l}\text { Chang et } \\
\text { coll., } 2012^{9}\end{array}$ & $\begin{array}{l}\text { - Effet inconstant sur l'IMC et le poids; } \\
\text { on a conclu que peu d'études } \\
\text { avaient quantifié cet effet } \\
\text { - Effet inconstant sur les niveaux de } \\
\text { l'activité physique (positif dans deux } \\
\text { cas et négatif dans trois cas) }\end{array}$ & $\begin{array}{l}\text { - Le taux d'utilisation des médias } \\
\text { sociaux était bas ( } 25 \% \text { ), et } 85 \% \text { des } \\
\text { études ont eu recours à des } \\
\text { plateformes élaborées par des } \\
\text { chercheurs et susceptibles de n'être } \\
\text { pas conviviales, dynamiques et liées } \\
\text { à une communauté importante } \\
\text { d'utilisateurs. } \\
\text { - L'utilisation des médias sociaux pour } \\
\text { la gestion du poids peut réduire les } \\
\text { impressions positives qui s'y } \\
\text { rattachent. }\end{array}$ & $\begin{array}{l}25 \% \text { des utilisateurs ont signalé } \\
\text { qu'ils se servaient de sites de médias } \\
\text { sociaux. }\end{array}$ & $\begin{array}{l}\text { «On a constaté que les médias } \\
\text { sociaux faisaient partie intégrante } \\
\text { d'interventions en ligne en gestion } \\
\text { du poids, en grande partie au } \\
\text { moyen de babillards et de } \\
\text { bavardoirs dont les avantages } \\
\text { étaient ambigus. » [TRADUCTION] }\end{array}$ \\
\hline $\begin{array}{l}\text { Eysenbach } \\
\text { et coll., } \\
2004^{34}\end{array}$ & $\begin{array}{l}\text { - Perte de poids ou maintien d'un } \\
\text { poids santé : Les résultats étaient } \\
\text { mixtes, mais peu importants en } \\
\text { majeure partie. } \\
\text { - Changement de comportement : Six } \\
\text { études ont porté sur ce résultat, } \\
\text { mais les taux d'abstinence ont été } \\
\text { plus élevés au chapitre du soutien } \\
\text { par les pairs dans une étude. Les } \\
\text { résultats ont été semblables au sein } \\
\text { du groupe bénéficiant d'une } \\
\text { intervention psychoéducative } \\
\text { complète. }\end{array}$ & $\begin{array}{l}\text { Aucun effet nuisible n'a été signalé. } \\
\text { Les auteurs ont constaté peu } \\
\text { d'intérêt du secteur commercial ou } \\
\text { du milieu professionnel à l'égard de } \\
\text { l'évaluation des communautés } \\
\text { virtuelles pures. Il est difficile de } \\
\text { recréer les études portant sur l'effort } \\
\text { autonome « naturel » dans les } \\
\text { établissements contrôlés, car les } \\
\text { participants peuvent avoir le désir } \\
\text { intrinsèque de faire partie de } \\
\text { communautés virtuelles, et le } \\
\text { recrutement général peut ne pas }\end{array}$ & $\begin{array}{l}\text { Certaines études ont conclu que la } \\
\text { composante des communautés } \\
\text { virtuelles n'était pas utilisée de } \\
\text { façon notable. }\end{array}$ & $\begin{array}{l}\text { «Compte tenu du grand écart sur } \\
\text { le plan des interventions, des outils } \\
\text { de mesure et des populations } \\
\text { ciblées dans les études ainsi que du } \\
\text { manque de rigueur } \\
\text { méthodologique dans la majorité } \\
\text { des études examinées, l'effet des } \\
\text { groupes de soutien en ligne sur les } \\
\text { résultats sanitaires et l'utilisation } \\
\text { des ressources en soins de santé } \\
\text { demeure ambigu. " } \\
\text { [TRADUCTION] }\end{array}$ \\
\hline
\end{tabular}

- Seulement trois études sur 12 portant sur la dépression et le soutien social ont signalé une amélioration.

- Les effets liés aux mesures de soutien social étaient mixtes : certaines études ont fait état d'effets importants, mais d'autres, non.

- Cinq études ont porté sur l'hémoglobine glycosylée, et seulement l'une d'elles a démontré une amélioration importante.

- Sur les trois études réalisées sur l'utilisation des soins de santé, une a indiqué une diminution importante, alors que deux ont démontré une augomentation du nombre d'appels téléphoniques à des fournisseurs.

Griffiths, - Trois essais multifactoriels sur quatre Aucun effet nuisible n'a été signalé. Non signalé $2009^{39} \quad$ ont signalé une réduction des symptômes de dépression, alors que l'autre n'a mentionné aucun effet.

- Les constatations des études non expérimentales étaient mixtes.

- Les études multifactorielles ont été beaucoup moins susceptibles d'entraîner des résultats positifs que les interventions autonomes.

- Le résultat n'a pas été influencé par l'utilisation de groupes de soutien synchrones sur Internet (bavardoirs) par rapport à celle de groupes de soutien asynchrones (babillards, serveurs de liste ou groupes de discussion), peu importe si l'étude a signalé ou non le recours aux services d'un modérateur ou si la plateforme était publique, axée sur la recherche ou à accès restreint. cerner la population concernée. [TRADUCTION]

« Il est essentiel que des recherches de haute qualité se penchent sur l'effet [des groupes de soutien sur Internet] sur les résultats liés à la dépression. » [TRADUCTION] 
TABLEAU 3 (suite)

Résultats des examens systématiques inclus

\begin{tabular}{ll} 
Référence & \multicolumn{1}{c}{ Résultats souhaitables } \\
\hline Hong et & • La plupart des études ont signalé \\
coll., & des effets positifs du soutien en \\
$2012^{35}$ & ligne, mais aucun des ECR n'a \\
& rendu compte de résultats positifs \\
& importants, p. ex. aucune \\
& amélioration de l'humeur, de \\
& l'adaptation au cancer, de l'état de \\
& santé autodéclaré et de la QV \\
& autodéclarée. \\
& - Un ECR sur quatre a signalé des \\
& améliorations sur le plan du bien- \\
& être émotionnel, mais les résultats \\
& liés au bien-être psychologique \\
& étaient mixtes (une étude a \\
indiqué des améliorations alors \\
qu'une autre a fait état d'une plus \\
grande détresse psychologique au \\
sein du groupe d'intervention).
\end{tabular}

Lai et coll., II n'y a aucune mesure quantitative des $2014^{31}$ avantages pour les idées suicidaires; deux articles ont fait part d'une rétroaction positive de la part des utilisateurs qui étaient en crise.

Medina Soutien mutuel, recherche de et coll., soutien dans le cadre d'un effort $2013^{32}$ autonome, apport d'un soutien en tant que devise mémorisée, modération de l'information,

- Le risque de qualité médiocre de l'information (dont seulement la moitié est fondée sur des éléments de preuve), les manquements possibles à l'obligation de confidentialité et les restrictions de l'accès peuvent ne pas correspondre au contexte culturel de l'utilisateur.

- Risque de message non authentique $(<5 \%$ au sein d'un groupe de soutien en ligne)

- Risque que l'effet de commentaires hostiles $s^{\prime}$ « enflamme »

- Risque de dépendance à Internet, qui se limite aux clients qui savent lire et écrire

- Préoccupations en matière de santé possibles lors de crises graves

- Insatisfaction ou mécontentement Non décrit par rapport à l'incapacité de trouver ce qu'on cherche

- Risque pour les personnes sensibles sur le plan social, risque d'absence d'une modération du contenu et risque d'obtention de données non fiables nombre de visites chez le médecin, diminution de l'isolement, amélioration de la QV (données et thèmes qualitatifs) et espoir accru

Moorhead - Interactions accrues avec les autres et coll., - Plus de renseignements $2013^{8}$ disponibles, échangés et personnalisés

- Accessibilité accrue et élargoissement de l'accès à I'information sur la santé

- Soutien par les pairs, soutien social et soutien affectif

- Surveillance de la santé publique

- Possibilité d'influencer la politique en matière de santé
Aucun effet nuisible signalé. Les limites comprenaient la fiabilité médiocre, les préoccupations en matière de qualité, l'absence de respect de la vie privée, l'ignorance des risques de la divulgation de renseignements, les conseils nuisibles ou inexacts, la surcharge de l'information, l'incertitude par rapport à la façon d'appliquer personnelles, la plus grande efficacité de certains médias quant l'information selon les situations
Non décrit

« Des éléments de preuve préliminaires tendent à indiquer l'avantage probable des stratégies Web pour la prévention du suicide. » [TRADUCTION]
Non décrit, mais certains écarts sur le plan de l'utilisation doivent être analysés plus à fond (p. ex. plus grand nombre d'utilisateurs de sexe féminin). Utilité au chapitre du soutien social et affectif, particulièrement en ce qui concerne l'établissement de contacts où les liens sont solides. Les interactions entre pairs et le déséquilibre ou le mauvais équilibre pour les professionnels dans les échanges entre professionnels et patients
« On a constaté des éléments de preuve préliminaires, mais non concluants par rapport aux résultats positifs. » [TRADUCTION]

« Les groupes de soutien en ligne désignent un vaste dépôt de renseignements de qualité sur les maladies générales et précises, comme les maladies

cardiovasculaires, ainsi qu'un environnement propice à la stimulation ou à la création d'une communauté de soutien chez les participants. » [TRADUCTION]

«Bien que le recours aux médias sociaux comporte des avantages pour la communication au sujet de la santé, l'information doit faire l'objet d'un contrôle aux fins de qualité et de fiabilité, et la confidentialité et le respect de la vie privée des utilisateurs doivent être maintenus. » [TRADUCTION]

Suite page suivante 
TABLEAU 3 (suite)

Résultats des examens systématiques inclus

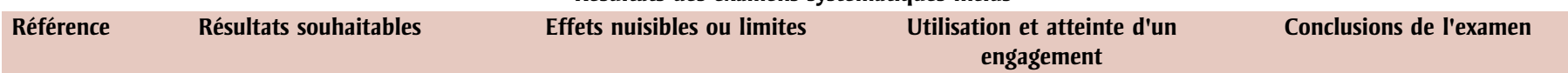

au changement de comportement,

Nef et L'avantage principal était de savoir coll., ce qui se passait dans la vie des plus $2013^{33} \quad$ jeunes membres de la famille.

Newton et On ne constate aucune différence Ciliska, sur le plan de l'effet dans $2006^{12} \quad$ l'inventaire des troubles alimentaires ou le questionnaire d'examen des troubles alimentaires.

Nieto,

$2008^{40}$

Les résultats n'ont pas été regroupés. Des résultats positifs ont découlé des deux études. les effets néfastes sur la santé, les comportements négatifs liés à la santé et la dissuasion de personnes de voir leurs professionnels de la santé.

Aucun effet nuisible à la santé mentale et aucune hausse de la solitude n'ont été signalés. Les obstacles décrits étaient les suivants : absence de respect de la vie privée, mauvaise compréhension de l'objet des sites de médias sociaux et nonconvivialité de la technologie.

Aucun effet nuisible ou aucune limite n'ont été signalés, mais les auteurs mentionnent qu'une préoccupation éthique par rapport aux interventions sur Internet se traduit par l'incapacité de détecter et de traiter les symptômes graves de troubles alimentaires qui pourraient être détectés lors d'une rencontre en personne.

Aucun effet nuisible n'a été signalé. Non signalé

Le taux de participation aux séances du groupe d'intervention a varié de $77 \%$ à $92 \%$.

L'acceptation et l'utilisation sont faibles. qualité des médias sociaux ne sont pas réglementées.
" Il est impossible de présenter des énoncés concluants au sujet des répercussions des programmes de prévention sur Internet. » [TRADUCTION]
Williams et Dans dix études, le poids n'est pas coll., $2014^{30}$ caractérisé par une différence importante sur le plan statistique : DMS : 0.00; IC à $95 \%$ : $-0,19$ à 0,19 . Les niveaux d'activité physique ne sont pas caractérisés par une différence importante sur le plan statistique : DMS : 0,13 ; IC à $95 \%$ : $-0,04$ à 0,30 ), 12 études.
Aucun effet nuisible n'a été signalé (mais le taux d'abandon du groupe d'intervention est élevé). Les auteurs ont fait état de certains risques pour la confidentialité, de différences culturelles, de commentaires hostiles, de faux messages et de préoccupations en matière de sécurité lors des crises.
$23 \%$ des membres du groupe d'intervention ont réalisé l'étude.

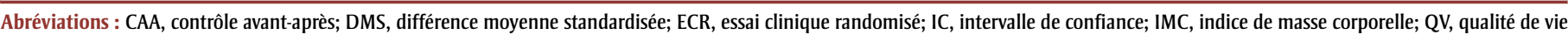

l'insatisfaction, les préoccupations au sujet du respect de la vie privée et la solitude. Newton et Ciliska ${ }^{12}$ ont relevé une préoccupation sur le plan éthique selon laquelle les interventions sur Internet pourraient ne pas entraîner la détection de symptômes graves qui auraient été détectés lors d'une rencontre en personne. Lai et coll. ${ }^{31}$ ont exprimé des préoccupations au sujet de la qualité des renseignements transmis aux utilisateurs des médias sociaux, notamment l'absence de confidentialité et l'incapacité des conseillers et des modérateurs à réagir en temps utile aux crises. Dans une analyse qualitative, Medina et coll. ${ }^{32}$ ont décrit le mécontentement ou l'insatisfaction des participants causés par un mauvais diagnostic ou un manque d'information ou de soutien, ce qui constitue une limite des médias sociaux. Nef et coll. ${ }^{33}$ (score de 3 à l'outil AMSTAR) ont constaté qu'aucun effet nuisible n'avait été signalé sur le plan de la santé mentale ou de la solitude, mais ils ont décrit les préoccupations des aînés par rapport au respect de la vie privée et au contenu inadéquat. Moorhead et coll. ${ }^{8}$ (98 études et score de 5 à l'outil AMSTAR) ont fait part de la fiabilité et de la qualité médiocres des renseignements sur les soins de santé ainsi que de l'absence de respect de la vie privée, qu'ils considèrent comme des effets nuisibles potentiels. Aucun des examens n'a dévoilé une augmentation des comportements négatifs ou malsains.
Quels éléments de preuve y avait-il par rapport à l'équité en matière de santé?

Les examens pris en compte n'ont présenté aucune analyse détaillée quant aux caractéristiques liées aux inconvénients. Trois examens ont semblé indiquer que les résultats pouvaient s'appliquer à différentes populations, selon les constatations qui y ont été faites, à savoir :

- Il n’y avait aucune différence entre les jeunes et les adultes sur le plan de l'efficacité des médias sociaux par rapport à l'alimentation saine, aux comportements et à l'activité physique ${ }^{30}$;

- Les participants qui avaient un capital social ou un soutien social de base 
moins importants étaient plus susceptibles d'utiliser les médias sociaux ${ }^{9}$;

- Les utilisateurs de médias sociaux provenaient, de façon disproportionnée, de ménages à revenu modique ${ }^{8}$.

Nef et coll. ${ }^{33}$ ont évalué des études qualitatives sur l'utilisation d'Internet par les aînés (personnes âgées de 55 ans et plus) et signalé qu'Internet les aidait à demeurer en contact avec leur famille et leurs amis, et qu'aucun élément de preuve n'indiquait que l'utilisation d'Internet avait entraîné chez eux une solitude accrue ou des effets nuisibles plus importants ${ }^{33}$. Dans leur examen, Lai et coll. $^{31}$ ont déclaré que la nature anonyme des activités sur Internet pouvait en fait contribuer à promouvoir un comportement favorisant la santé au sein des groupes à risque plus difficiles à rejoindre. Hong et coll. ${ }^{35}$ ont affirmé que les médias sociaux favorisaient le contact avec les participants de régions rurales (bien qu'une seule étude ait pris en compte des populations de régions rurales). Deux examens ont signalé que les niveaux d'alphabétisation pouvaient influer sur l'efficacité de l'intervention ${ }^{31,35}$. Seuls Moorhead et coll. ${ }^{8}$ ont mentionné qu'il était possible d'adapter les documents transmis dans les médias sociaux en fonction des niveaux d’alphabétisation.

\section{Quels éléments conceptuels font la} promotion de l'équité en matière de santé?

À l'aide de l'EIES du MSSLD, nous avons décrit les préoccupations potentielles de douze populations, notamment les communautés ethnoraciales, les personnes de catégories d'âge différentes, les personnes de sexe et de genre différents et les personnes atteintes d'une incapacité physique. Dans le cas des études visées par les examens systématiques qui faisaient référence aux stratégies d'atténuation potentielles, nous avons décrit les stratégies proposées.

La principale préoccupation touchant plusieurs populations est la possibilité d'un accès restreint à Internet (p. ex. sans-abri ou aînés à faible revenu). Pour atténuer ces inégalités, la promotion de la santé à l'aide des médias sociaux peut nécessiter la prestation d'un accès à Internet ainsi qu'à des ordinateurs ou à des appareils mobiles sans fil.
Aucun des examens cités n'a fait mention de ressources supplémentaires susceptibles de s'imposer pour l'apport des changements de comportement préconisés par les interventions de médias sociaux, notamment la présence de sentiers pédestres, l'accès à des centres de conditionnement physique, le caractère abordable des co-interventions (comme des timbres de nicotine pour les gens qui veulent cesser de fumer), l'accès à des aliments abordables et l'accès à des soins de santé. Par contre, ces déterminants et facilitateurs des changements de comportement doivent être pris en considération dans le cadre de la théorie qui sous-tend le programme et en vue de l'établissement de toute mesure d'intervention dans les médias sociaux $^{36}$.

Étant donné que les nouvelles interventions dans les médias sociaux s'appuient sur le réseau des individus qui en font partie, les personnes qui disposent de réseaux sociaux faibles peuvent être désavantagées (p. ex. aînés à faible revenu qui ont peu de liens hormis avec leurs petitsenfants $^{33}$ ). Cependant, Chang et coll. ${ }^{9}$ ont démontré que les personnes ayant des réseaux sociaux faibles avaient tendance à utiliser davantage les médias sociaux, ce qui donne à penser qu'elles pourraient bénéficier de ces interventions.

Plusieurs examens ont soulevé la préoccupation de l'acceptabilité des interventions dans les médias sociaux. L'acceptabilité peut se rattacher à l'acceptabilité et aux normes culturelles (notamment dans le cas d'une étude destinée à un auditoire qui parle hébreu ${ }^{31}$ ) ou aux préférences propres à une population (p. ex. un examen des aînés a démontré que ceux-ci ne connaissaient pas bien Facebook ${ }^{33}$ ). Selon plusieurs examens, un essai par les utilisateurs et un test d'acceptabilité contribueraient à accroître la probabilité que les populations cibles s'adonnent aux interventions dans les médias sociaux.

Les examens ont signalé que les préoccupations relatives au respect de la vie privée et la confidentialité pouvaient représenter un problème pour certaines populations, comme les aînés, et qu'elles pouvaient influer sur le recours aux interventions dans les médias sociaux. Le contrôle de la qualité concernant les sites de médias sociaux, comme celui d'un modérateur, pourrait contribuer à réduire les préoccupations liées au respect de la vie privée et à encourager l'utilisation des médias sociaux ${ }^{8}$.

Certaines populations peuvent être particulièrement sensibles aux commentaires hostiles ou trompeurs. Dans l'affirmative, pour ces interventions, un modérateur pourrait pouvoir limiter l'accès au groupe de médias sociaux afin de réduire le risque d'utilisation inadéquate.

\section{Secteurs propices à l'approfondissement de la recherche}

On n'a trouvé aucun exemple d'examen systématique qui était axé sur l'utilisation d'applications pour téléphones intelligents et tablettes et des médias sociaux pour la promotion de la santé, probablement parce que les applications pour téléphones intelligents n'ont été mises au point et à l'essai que récemment. Une conception conviviale a été décrite comme un secteur à améliorer.

Un secteur propice à l'approfondissement de la recherche est le recours aux plateformes de médias sociaux bien connues (comme Facebook et Twitter) pour les interventions en promotion de la santé. Seulement 28 études (abordées dans quatre examens) étaient axées sur l'utilisation de sites de médias sociaux bien connus en tant que plateforme pour l'intervention; dans le cas des autres études, on s'était servi de plateformes conçues par les chercheurs. Ces plateformes fermées n'ont pas tiré profit des réseaux sociaux en place des participants, mais elles visaient plutôt à établir des liens entre les utilisateurs du même programme de promotion de la santé et de recherche de soutien, de sorte que ceux-ci puissent faire part de leur expérience.

\section{Analyse}

Les examens recueillis dans cette synthèse semblent indiquer qu'il y a un potentiel sur le plan de l'amélioration des résultats sanitaires et des comportements liés à la promotion de la santé au sein des populations ciblées. Toutefois, les inégalités accrues en matière de santé constituaient également un risque, en raison des problèmes touchant l'accès aux médias sociaux, l'acceptabilité 
de ces derniers et l'absence de surveillance de leur qualité.

Les travaux de recherche à venir doivent viser à déterminer les interventions dans les médias sociaux qui sont efficaces et à décrire l'ensemble de leurs spécificités, notamment en ce qui concerne la façon dont elles sont exécutées et utilisées, grâce à des critères explicites comme la liste de vérification TIDIER (Template for Intervention Description and Replication) ${ }^{37}$. Les travaux de recherche doivent également documenter de façon explicite les comportements négatifs accrus, la stigmatisation ou l'aggravation des inégalités en matière de santé si certaines populations sont exclues.

Les interventions qui ont recours aux sites de médias sociaux les plus connus peuvent être caractérisées par des taux d'utilisation et d'acceptabilité plus élevés parce qu'elles tirent profit des réseaux sociaux en place. Ces plateformes doivent faire l'objet d'une évaluation dans le cadre de travaux de recherche à venir.

On n’a relevé aucune analyse détaillée des caractéristiques liées aux inconvénients. Certaines études ciblées ont toutefois signalé que les interventions dans les médias sociaux étaient efficaces chez les jeunes et les aînés ${ }^{30,33}$, au sein des groupes ayant un statut socioéconomique plus faible ${ }^{8}$, chez les personnes de différentes ethnicities ${ }^{31}$ et chez les participants des régions rurales ${ }^{35}$. Plusieurs examens ont semblé indiquer que les médias sociaux pourraient faciliter l'engagement des populations plus difficiles à rejoindre et viser à surmonter les lacunes sur le plan de l'alphabétisation et des études, pour autant qu'il y a un accès à la technologie. Par contre, on disposait de très peu d'éléments de preuve pour ces populations dans les études primaires ou les examens systématiques. Les examens systématiques et les études primaires à venir doivent donc être axés sur la collecte de données et l'analyse de l'effet de l'intervention pour différents groupes de population.

Il est important d'effectuer une recherche qualitative sur le rôle de la conception et de l'évaluation de programmes fondés sur la théorie, l'utilisation de nombreuses composantes, la conception axée sur l'utilisateur ainsi que la mesure du processus d'exécution (notamment l'utilisation, l'interaction et la satisfaction). Au moment de planifier une intervention dans les médias sociaux, il est important de prendre en compte l'utilisation de base par la population cible des médias sociaux. Les médias sociaux permettent d'établir un contact avec les populations plus difficiles à rejoindre étant donné que l'accès à Internet est offert à grande échelle au Canada.

\section{Points forts et limites}

L'un des points forts de la présente vue d'ensemble est l'adoption d'une approche élargie qui permet d'examiner les effets de diverses interventions dans les médias sociaux pour un large éventail d'états de santé. Malgré les critères larges d'inclusion, nous avons seulement trouvé des études qui comparaient les conditions d'intervention minimales (p. ex. accès à des versions non interactives ou limitées des sites Web d'études) ${ }^{30}$. Nous avons eu recours à des méthodes d'analyse rapide pour répondre au besoin établi par notre utilisateur des connaissances. La qualité de l'établissement des rapports dans les examens systématiques a réduit notre éventail : 5 des 11 examens avaient un score de 5 ou moins sur une possibilité de 11 à l'outil AMSTAR pour la qualité en raison des limites associées à la stratégie de recherche, aux critères d'admissibilité et à la documentation des résultats. Autrement dit, presque la moitié des examens systématiques étaient de faible qualité. Un seul examen a rendu compte de l'ampleur de l'effet ainsi que des intervalles de confiance (IC), les autres ayant présenté un résumé descriptif ou n'ayant indiqué que les valeurs $p$. Ce fait a limité la capacité d'évaluer l'ampleur des effets et la confiance à l'égard des estimations.

Dans le cadre des examens systématiques, il n'y a pas eu de contrôle ou de compte rendu réguliers de l'utilisation des interventions dans les médias sociaux. Seulement quatre examens ont rendu compte de l'utilisation par les participants de l'intervention, tous les examens ayant indiqué une faible utilisation ${ }^{8,9,30,34}$. Plus de $20 \%$ des participants ont abandonné les études, et un examen a signalé que moins de $25 \%$ des participants avaient eu recours aux médias sociaux offerts dans le cadre des études ${ }^{9}$. Par conséquent, il est impossible de déterminer si l'absence d'effets, incluant les effets mixtes, a été causée par l'absence d'utilisation des médias sociaux par les participants ou par l'inefficacité des médias sociaux. Les effets mixtes peuvent également s'expliquer par des facteurs comme les différences touchant les populations, la conception et l'exécution des interventions ainsi que les interventions concomitantes.

L'EIES présente une approche structurée visant à évaluer les effets prévisibles comme imprévisibles, ce qui contribue à l'établissement de stratégies d'atténuation. Idéalement, cet outil sert plutôt à éclairer la planification et l'élaboration d'interventions, et la consultation et l'engagement avec les communautés touchées y sont essentiels $^{38}$.

Enfin, on a eu recours à une approche de synthèse rapide et, par conséquent, on a effectué un tri dans les examens définis dans le cadre de nos activités de recherche et d'extraction de données, l'évaluation de la qualité ayant été assurée par un auteur. Cette limite n'a selon nous pas d'incidence sur les résultats de notre synthèse.

\section{Conclusion}

Sur la base de notre synthèse des examens systématiques, nous pouvons affirmer que les effets des interventions dans les médias sociaux sont mixtes, voire négligeables. On dispose de suffisamment d'éléments de preuve des caractéristiques de conception et d'exécution (p. ex. intensité et durée des interventions) susceptibles d'entraîner une amélioration des effets. Il faut réaliser plus de travaux de recherche sur les médias sociaux en mobilisant ceux qui sont en place (plutôt que les plateformes qui permettent de faire uniquement de la recherche) et tenir compte de l'acceptabilité et de l'utilisation des médias sociaux ainsi que de l'évaluation des effets désirables et indésirables.

\section{Remerciements}

Ce projet a été financé par l'Agence de la santé publique du Canada. 


\section{Références}

1. Neiger BL, Thackeray R, Van Wagenen SA, Hanson CL, West JH, Barnes MD et collab. Use of social media in health promotion: purposes, key performance indicators, and evaluation metrics. Health Promot Pract. 2012;13(2):159-164.

2. Agence de la santé publique du Canada. Santé publique ASPC [Internet]. @ASPC_ GC2015 [consulté le 17 janvier 2016]. Consultable à partir du lien. https://twitter. com/aspc_gc.

3. Agence de la santé publique du Canada. Public Health Agency of Canada. Agence gouvernementale [Internet]. Menlo Park (CA) : Facebook; 2015 [consulté le 17 janvier 2016]. Consultable à la page. https://www.facebook.com/Public-Health-Agency-of-Canada10860597051/timeline/

4. Davies J, Mai D, Williams M, Dhaliwal M, Brankley L, McColl K. Social Media and Technology Project Team. Environmental scan of social media at Ontario Public Health Units: analyzing the effectiveness of social media planning, implementation and evaluation at Health Units in Ontario. Guelph (Ont.) : Wellington-Dufferin-Guelph Public Health; 2013.

5. Lenhart A, Purcell K, Smitth A, Zickuhr K. Social media and young adults [Internet]. Washington (DC) : Pew Research Center; 2010Feb3[consulté le 14 janvier 2015]. Consultable à la page. http://www.pewinternet.org/2010/02/03/social-media-andyoung-adults/

6. Statistique Canada. Tableau 358-0153. Enquête canadienne sur l'utilisation d'Internet, utilisation d'Internet, selon le groupe d'âge, l'activité sur Internet, le sexe, le niveau de scolarité et le revenu du ménage, occasionnel (pourcentage) [Internet]. Ottawa (Ont.) : CANSIM database; [modifié le 28 octobre 2013; consulté le 4 novembre 2014]. Consultable à la page: http://www5.statcan.gc. $\mathrm{ca} /$ cansim/a26? id $=3580153$ \&retrLang $=$ fra\&lang $=$ fra

7. Kietzmann $\mathrm{JH}$, Hermkens $\mathrm{K}$, McCarthy IP, Silvestre BS. Social media? Get serious! Understanding the functional building blocks of social media. Bus Horizons. 2011;54(3):241-251.
8. Moorhead SA, Hazlett DE, Harrison L, Carroll JK, Irwin A, Hoving C. A new dimension of health care: systematic review of the uses, benefits, and limitations of social media for health communication. J Med Internet Res. 2013;15(4):e85. DOI: 10.2196/jmir.1933.

9. Chang T, Chopra V, Zhang C, Woolford SJ. The role of social media in online weight management: systematic review. J Med Internet Res. 2013;15(11):e262. DOI: 10.2196/jmir.2852.

10. Bailey JV, Murray E, Rait G et collab. Computer-based interventions for sexual health promotion: systematic review and metaanalyses. Int J STD AIDS. 2012;23(6): 408-413. DOI: 10.1258/ijsa.2011.011221.

11. Murray E, Burns J, See TS, Lai R, Nazareth I. Interactive health communication applications for people with chronic disease. Cochrane Database Sys Rev. 2005(4):CD004274.

12. Newton MS, Ciliska D. Internet-based innovations for the prevention of eating disorders: a systematic review. Eat Disor. 2006;14(5):365-384.

13. Lorenc T, Petticrew M, Welch V, Tugwell P. What types of interventions generate inequalities? Evidence from systematic reviews. J Epidemiol Community Health. 2013;67(2): 190-193. DOI: 10.1136/jech-2012-201257.

14. Whitehead M. The concepts and principles of equity and health. Int J Health Services. 1992;22(3):429-445.

15. O'Connor D, Green S, Higgins JP. Chapter 5: Defining the review question and developing criteria for including studies. Dans Higgins JP, Green S (dir.) Cochrane handbook of systematic reviews of intervention, Cochrane Book Series Collaboration. Hoboken (NJ) : John Wiley and Sons; 2011.

16. Meglic M, Lamut A, Sinkovec A, Yared W. Chapter 3: Online social media as a tool to improve cancer prevention and health promotion. Dans Martin-Moreno JM, Albreht T, Rados Krnel S (dir.) Boosting innovation and cooperation in European cancer control: key findings from the European Partnership for Action Against Cancer. Ljubljana : National Institute of Public Health of the Republic of Slovenia; Brussels: World Health Organization on behalf of the European Observatory on Health Systems and Policies; 2013.
17. Sterne J. Social media metrics: how to measure and optimize your marketing investment. Hoboken (NJ) : John Wiley and Sons; 2010.

18. Black AD, Car J, Pagliari $\mathrm{C}$ et collab. The impact of eHealth on the quality and safety of health care: a systematic overview. PLoS Med. 2011;8(1):e1000387. DOI: 10.1371/journal. pmed.1000387.

19. French B, Day E, Watkins C et collab. The challenges of implementing a telestroke network: a systematic review and case study. BMC Med Inform Decis Mak. 2013;13:125. DOI: $10.1186 / 1472-6947-13-125$.

20. Jackson N, Waters E. Guidelines for systematic reviews in health promotion and public health taskforce. Criteria for the systematic review of health promotion and public health interventions. Health Promot Int. 2005;20(4):367-374.

21. O'Neill J, Tabish H, Welch V et collab. Applying an equity lens to interventions: using PROGRESS ensures consideration of socially stratifying factors to illuminate inequities in health. J Clinical Epidemiology. 2014;67(1): 56-64. DOI: 10.1016/j.jclinepi.2013.08.005.

22. Khangura S, Konnyu K, Cushman R, Grimshaw J, Moher D. Evidence summaries: the evolution of a rapid review approach. Syst Rev. 2012;1:10. DOI: 10.1186/2046-40531-10.

23. Hartling L, Guise JM, Kato E et collab. EPC Methods: an exploration of methods and context for the production of rapid reviews. Research White Paper. Rockville (MD) : Agency for Healthcare Research and Quality; 2015.

24. Montori VM, Wilczynski NL, Morgan D, Haynes RB. Optimal search strategies for retrieving systematic reviews from Medline: analytical survey. BMJ. 2005;330(7482):68.

25. Sampson M, McGowan J, Lefebvre C, Moher D, Grimshaw J. PRESS: peer review of electronic search strategies. Ottawa (Ont. ) : Agence canadienne des médicaments et des technologies de la santé; 2008.

26. Shea BJ, Hamel C, Wells GA et collab. AMSTAR is a reliable and valid measurement tool to assess the methodological quality of systematic reviews. J Clin Epidemiol. 2009;62(10): 1013-1020. DOI: 10.1016/j.jclinepi.2008.10.009. 
27. Welch V, Brand K, Kristjansson E, Smylie J, Wells G, Tugwell P. Systematic reviews need to consider applicability to disadvantaged populations: inter-rater agreement for a health equity plausibility algorithm. BMC Med Res Method. 2012;12:187. DOI: 10.1186/ 1471-2288-12-187.

28. Ministry of Health and Long-Term Care. Health rquity impact assessment (HEIA) workbook. Toronto (ON) : Ontario Ministry of Health and Long-Term Care; 2012.

29. Moher D, Liberati A, Tetzlaff J, Altman DG. Preferred reporting items for systematic reviews and meta-analyses: the PRISMA statement. BMJ. 2009;339:b2535.

30. Williams G, Hamm MP, Shulhan J, Vandermeer B, Hartling L. Social media interventions for diet and exercise behaviours: a systematic review and meta-analysis of randomised controlled trials. BMJ Open. 2014;4(2):e003926.

31. Lai MH, Maniam T, Chan LF, Ravindran AV. Caught in the web: a review of web-based suicide prevention. J Med Internet Res. 2014;16(1):e30. DOI: 10.2196/jmir.2973.

32. Medina EL, Loques Filho O, Mesquita CT. Health social networks as online life support groups for patients with cardiovascular diseases. Arq Bras Cardio. 2013;101(2):e39-45.

33. Nef T, Ganea RL, Muri RM, Mosimann UP. Social networking sites and older users - a systematic review. Int Psychogeriat. 2013; 25(7):1041-1053.

34. Eysenbach G, Powell J, Englesakis M, Rizo C, Stern A. Health related virtual communities and electronic support groups: systematic review of the effects of online peer to peer interactions. BMJ. 2004;328(7449): 1166.

35. Hong Y, Pena-Purcell NC, Ory MG. Outcomes of online support and resources for cancer survivors: a systematic literature review. Patient Educ Couns. 2012;86(3): 288-96. DOI: 10.1016/j.pec.2011.06.014.

36. Webb TL, Joseph J, Yardley L, Michie S. Using the internet to promote health behavior change: a systematic review and metaanalysis of the impact of theoretical basis, use of behavior change techniques, and mode of delivery on efficacy. J Med Internet Res. 2010;12(1):e4. DOI: 10.2196/jmir.1376.
37. Hoffmann TC, Glasziou PP, Boutron I, Milne $\mathrm{R}$, Perera R, Moher D et collab. Better reporting of interventions: template for intervention description and replication (TIDieR) checklist and guide. BMJ. 2014;348: g1687. DOI: 10.1136/bmj.g1687.

38. Povall SL, Haigh FA, Abrahams D, ScottSamuel A. Health equity impact assessment. Health Promot Int. 2014;29(4): 621-33. DOI: $10.1093 /$ heapro/dat012.

39. Griffiths KM, Calear AL, Banfield M. Systematic review on Internet support groups (ISGs) and depression (1): do ISGs reduce depressive symptoms?. J Med Internet Res. 2009;11(3):e40. DOI: 10.2196/jmir.1270.

40. Nieto R, Miro J, Huguet A. [New information and communication technologies in the treatment of chronic pain]. Rev Esp Anestesiol Reanim. 2008;55:426-433. 